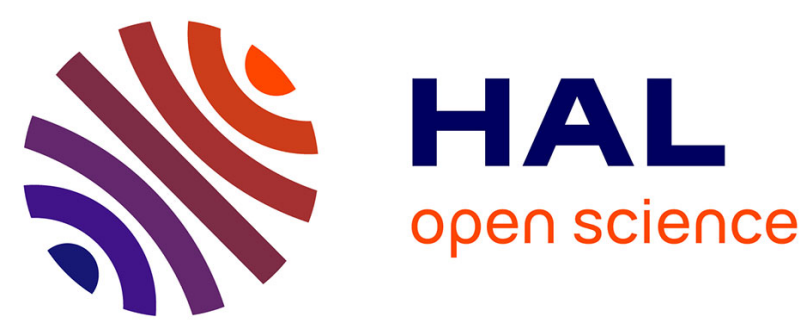

\title{
La montée en puissance des territoires : facteur de recomposition ou de décomposition des politiques culturelles?
}

\author{
Emmanuel Négrier, Philippe Teillet
}

\section{To cite this version:}

Emmanuel Négrier, Philippe Teillet. La montée en puissance des territoires : facteur de recomposition ou de décomposition des politiques culturelles?. Jean-Pierre Saez. Culture et société: un lien à recomposer, Saison une - Collections - 65, Éditions de l'Attribut, pp.91-108, 2008, 978-2-916002-08-8. hal-01444175

\author{
HAL Id: hal-01444175 \\ https://hal.science/hal-01444175
}

Submitted on 23 Jan 2017

HAL is a multi-disciplinary open access archive for the deposit and dissemination of scientific research documents, whether they are published or not. The documents may come from teaching and research institutions in France or abroad, or from public or private research centers.
L'archive ouverte pluridisciplinaire HAL, est destinée au dépôt et à la diffusion de documents scientifiques de niveau recherche, publiés ou non, émanant des établissements d'enseignement et de recherche français ou étrangers, des laboratoires publics ou privés. 


\title{
Emmanuel Négrier, Philippe Teillet
}

\section{La montée en puissance des territoires : facteur de recomposition ou de décomposition des politiques culturelles ?}

\author{
dans J.P.Saez (dir), Culture et Société, Paris : Éditions de l’Attribut, 2008, p.91-108
}

Dans différents pays (Bonet, Négrier, 2007), les politiques culturelles sont de moins en moins marquées par un pilotage et des enjeux nationaux. En revanche, elles s'organisent plus souvent territoires par territoires (Saez, 2004). En France, ce fait est fréquemment vécu comme une menace. Désengagement de l'Etat, dirigisme des élus locaux, rupture d'égalité entre les citoyens, sont les termes principaux d'une polémique qui se développe de façon récurrente autour de cette évolution sans pour autant parvenir à la freiner.

Le renforcement des autorités locales et des pouvoirs urbains en particulier, poussé par l'urbanisation croissante de la population mondiale, est un phénomène qui dépasse largement nos frontières. La constitution de structures politiques supranationales (I'Union européenne, notamment) vient simultanément disputer par le haut le pouvoir régulateur des Etats centraux sur leur propre territoire. Enfin, l'émergence ou le développement de sentiments d'appartenance non nationaux, fondés sur des différences culturelles multiples (régionales, ethniques, religieuses, sexuelles ou esthétiques), signale l'épuisement du long processus de construction des identités nationales. Les politiques publiques de la culture, qui avaient apporté une contribution essentielle à ce processus, sont appelées à s'adapter à ce nouveau contexte. De ce point de vue, le modèle français de politiques culturelles est plus que d'autres mis au défi par ce que Luis Bonet et Emmanuel Négrier ont appelé « la fin des cultures nationales » (Bonet, Négrier, 2007). L'approche républicaine de la citoyenneté a en effet longtemps donné à l'action culturelle son ossature politique et sa raison d'être. A l'inverse, sa fragmentation territoriale et son frémissement multiculturaliste la privent d'une partie de ses fondements idéologiques traditionnels. Plus encore, les modes de consécration culturelle sont restés centralisés, conférant en la matière, à la scène parisienne et aux médias nationaux, un rôle clef. Cette rupture entre espaces de production et de consécration ne peut qu'inquiéter les professionnels de la culture inscrits dans des «mondes » dépassant les frontières territoriales de ceux qui sont désormais leurs principaux financeurs.

Ce sont toutes ces raisons qui nous ont conduits à examiner de près les termes du débat portant sur la territorialisation des politiques culturelles. La perspective grisante de transformation d'un modèle d'action dont les limites sont aujourd'hui sensibles, va de pair avec les craintes que nourrissent les incertitudes sur l'avenir des politiques publiques en ce domaine. Entre recomposition et décomposition, l'impact de logiques territoriales dans le champ des politiques culturelles se dessine pour l'instant avec difficulté.

\section{Ce que territorialiser veut dire}

Territorialiser, c'est donner un sens politique à l'espace. Au cours du temps, le sens originel du mot «territoire » (I'un de ses racines, en droit canon, est le « jus terrendi »: le droit de terrifier) s'est transformé en celui d'espace légitime du pouvoir. Il existe donc un lien consubstantiel entre la notion de territoire et la dimension politique des problèmes. De son côté, la territorialisation est un processus permanent 
qui a connu des cycles différents. Des courants d'analyse successifs ont tenté d'en donner la représentation la plus pertinente.

\section{Le territoire entre verticalité et horizontalité}

Il y a trente ou quarante ans, le territoire était pensé à partir d'une vision assez homogène et centraliste rassemblant autant les juristes les plus positivistes (avec leur notion de puissance publique) que les sociologues (avec celle de pouvoir périphérique $^{1}$ ). Jusque vers 1985 , la notion en vogue a été celle de « régulation croisée ». L'examen des relations entre les préfets et les notables montrait en effet que la cohérence d'une société sur un territoire donné résultait simultanément de l'influence de normes centrales et d'un compromis localisé pour les « accommoder». Autrement dit, on privilégiait alors une approche verticale des problématiques territoriales, avec des corrections « dialectiques» prenant en compte, mais à la marge, la parole et le rôle des acteurs locaux dans une perspective que l'on pourrait qualifier d'horizontale (pour continuer de filer la métaphore géométrique dans l'analyse des jeux des pouvoirs).

À la fin des années quatre-vingt, ce courant d'analyse entre en crise du fait de la décentralisation et de la multiplication des dissonances locales vis-à-vis de ce modèle théorique. L'approche des territoires s'inverse progressivement pour devenir une pensée à dominante presque horizontale avec quelques accommodements verticaux. Les notions qui émergent alors sont celles de gouvernance territoriale, de gouvernement urbain, d'échange politique territorial. Il est devenu nécessaire de comprendre la territorialisation pour prendre la mesure de la cohérence ou de

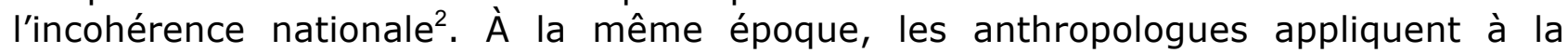
société française les mêmes méthodes d'étude qu'aux civilisations premières et restituent ainsi l'épaisseur territoriale qui manque à l'analyse classique des élections ${ }^{3}$.

La réflexion territoriale s'est aussi ouverte à des travaux étrangers : la théorie de la gouvernance est très fortement influencée par les études anglo-saxonnes; l'analyse du développement économique localisé subit l'influence de la sociologie italienne du régionalisme économique, dont témoignent les travaux de Carlo Trigilia ${ }^{4}$. La réflexion sur le territoire est aujourd'hui au croisement d'une tradition (jacobine) privilégiant la dimension verticale des enjeux territoriaux, et de multiples pistes privilégiant aujourd'hui les relations ou causalités plus horizontales. Mais pour être plus sensible à la qualité des espaces, l'analyse des enjeux de pouvoir et de politique publique doit nécessairement sortir du travers «localiste ». Cela est particulièrement vrai si on considère que le territoire est non seulement le lieu d'une expression spécifique mais aussi une échelle de comparaison («benchmarking » territorial pour les Anglo-saxons). La territorialisation est aussi la capacité de se projeter à une échelle plus importante.

\footnotetext{
${ }^{1}$ Pierre Grémion, Le Pouvoir Périphérique, Seuil 1976.

2 Pour une application de cette idée à l'analyse des partis politiques, cf. F. Sawicki, Les réseaux du parti socialiste Belin, 2000. Quant à l'analyse des politiques publiques, cf. A. Faure, R. Balme et A. Mabileau, Les nouvelles politiques locales, Presses de Science Po, 1998.

3 cf. Yves Pourcher, Les maîtres de granit Omnibus, 1995.

${ }^{4}$ Cf. (avec C. Crouch, P. Le Galès, H. Voelzkow), Changing Governance of local economies: Responses of European Local Production Systems, Oxford, Oxford University Press, 2004 ; (avec M. Oberti), Sociologie économique, Armand Colin, 2002.
}

Page 2 sur 10 
Cette question renvoie aujourd'hui à des changements récents dont les mieux identifiables sont rythmés par la production législative (lois Voynet et Chevènement de 1999) et par la deuxième décentralisation consécutive à la loi du 13 août 2004). Les moins visibles sont la professionnalisation de l'action publique territoriale et le fait que les pouvoirs locaux se dotent de plus en plus d'instruments d'action publique très semblables à ceux de l'État.

\section{La territorialisation entre promesses et ambivalences}

La territorialisation met tout d'abord en valeur des caractères jusque-là occultés des territoires: leur culture politique singulière, leur attractivité propre, leur génie particulier en matière de gouvernance par exemple. Ces traits spécifiques sont mis au service de la volonté d'atteindre des buts collectifs et de produire des biens publics, c'est-à-dire de pratiquer une gouvernance territoriale visant à assurer un rayonnement territorial et à construire des formes de solidarité plus satisfaisantes dans ces territoires. Cela apparaît tout particulièrement dans l'intercommunalité. La territorialisation doit aussi permettre une proximité démocratique en rapprochant la décision du citoyen et en faisant coïncider territoire électoral et territoire des problèmes. Dans le domaine de la culture, les politiques de lecture publique, les politiques archivistiques départementales (à l'instar des politiques régionales de construction des lycées) démontrent depuis vingt ans que la territorialisation permet de construire l'action publique sur des bases plus intéressantes que dans un contexte centralisé. Il reste cependant encore beaucoup à faire pour démocratiser l'action publique. La décision s'est rapprochée du citoyen sans véritablement se démocratiser même si les élus actuels ont une sensibilité à la construction locale des problèmes qui diffère de celle des préfets avant la décentralisation.

Toutefois, ces promesses ne doivent pas occulter un certain nombre d'ambivalences. Celles-ci résident pour une part dans les motivations des acteurs qui participent aux formes de territorialisation ou qui les enclenchent: pourquoi l'État décentralise-t-il un certain nombre de compétences ? Agit-il au nom d'un principe d'efficacité supérieure, de démocratisation ou se défausse-t-il sur les pouvoirs locaux de compétences qu'il estime trop coûteuses pour lui ? La même question se pose à propos de la dynamique intercommunale, entre communes et intercommunalité. Il existe aussi une vraie ambivalence sur le fond: territorialiser c'est accepter des différences, mais jusqu'à quel point cela permet-il de supporter des inégalités ? C'est le dilemme classique de savoir à quel niveau juger des inégalités: la région, le département, l'agglomération ? La manière de construire un schéma territorial illustre particulièrement bien ce dilemme, dans la culture comme dans bien d'autres domaines de l'action publique. C'est pourquoi, la territorialisation ne doit pas être le principe unique de I'action publique : territorialiser suppose au contraire des formes de régulation à l'allemande, c'est-à-dire la mise en œuvre d'un principe, beaucoup plus effectif qu'en France, de péréquation entre territoires. Il existe donc une ambivalence entre la territorialisation et les principes plus généraux de l'action publique (égalité, redistribution) qui alimente le débat sur la place de nouveaux instruments : l'évaluation, la péréquation, la discrimination positive. Une dernière ambivalence réside enfin dans la perception que les agents ont des mérites ou des menaces que font peser sur eux les démarches territorialisantes. De ce point de vue, le secteur des politiques culturelles est un terrain particulièrement riche pour observer ces tensions entre bénéficiaires et victimes de la territorialisation (du moins entre ceux qui se projettent plutôt dans I'une ou dans l'autre de ces deux catégories). On peut même 
observer des situations de déchirements internes (à des groupes ou à des individus) tiraillés entre espoirs et craintes.

\section{Ce que cache la territorialisation culturelle}

En matière de politique culturelle, le double processus de décentralisation et de déconcentration a nettement changé la donne. Mais, dans ce champ d'intervention caractérisé depuis le début des années 60 par le rôle dominant du pouvoir central (souvent présenté, en outre, comme un héritage d'Ancien régime), les transformations sont telles que, symétriquement, les craintes et les résistances à l'encontre de ces évolutions ont été plus fortes et plus marquées ici que dans d'autres secteurs. Ces tensions peuvent être examinées au niveau de trois catégories d'acteurs: les services ministériels de la Culture, les professionnels de la culture et les collectivités territoriales.

La territorialisation vue du côté des services de l'Etat et des professionnels de la culture

Le ministère de la Culture a géré la décentralisation des années 1982-1983 et la déconcentration à partir de 1992. Il a accompagné le renforcement de la décentralisation, même si elle n'a été évoquée dans ses missions officielles qu'à partir de 2002. Le ministère est donc désormais chargé d'accompagner ce mouvement général pour ce qui le concerne. Il est devenu un partenaire des acteurs territoriaux avec ses services « de proximité » que sont les Directions régionales des affaires culturelles (DRAC), même si les dernières orientations de sa politique passent cette question totalement sous silence ${ }^{5}$. La déconcentration des crédits d'intervention est aujourd'hui une réalité. Ce ministère a en outre été à la pointe de la réflexion sur de nouveaux modes de gestion de l'action publique avec des tests sur des procédures nouvelles bien qu'un peu oubliées aujourd'hui, comme les protocoles de décentralisation à partir de 2000 et les expérimentations régionales de dispositifs territoriaux en 2003.

Cependant, son action en faveur de la territorialisation trouve assez vite ses limites. Ses moyens d'action (financiers et humains) n'ont pas été véritablement ou suffisamment augmentés, ce qui concrètement affecte sensiblement sa légitimité aux yeux de ses partenaires territoriaux. De plus, la loi organique relative aux lois de finances du 1er août 2001 (LOLF) se traduit par une forme de recentralisation : elle impose à partir du centre une définition a-territoriale des missions, des programmes d'action, ainsi que des indicateurs de performance. Il faut aussi rappeler les difficultés de tous les ministères à concevoir et assumer la différenciation des territoires. Alors que la politique contractuelle (lancée au milieu des années 70 puis à nouveau au cours des années 80 ) était potentiellement productrice de différences, le ministère de la culture s'en est plutôt servi comme d'un outil d'égalisation entre les territoires (fonction latente des conventions de développement culturel, en particulier). Ajoutons que le grand « récit » des agents de ce ministère consistait à lui donner pour mission d'abstraire les individus de leur culture d'appartenance afin de les faire accéder à une forme d'universalité. Cette conception, quasi identitaire pour ces personnels d'État, est toujours présente chez nombre d'entre eux. De même, l'idée d'une fonction égalisatrice de l'action de l'État, chargé de favoriser partout une égalité d'accès à la

\footnotetext{
${ }^{5}$ Voir la lettre de mission du président de la République à la ministre de la culture, du $1^{\text {er }}$ août 2007 qui se contente d'évoquer le seul rééquilibrage de son action entre le territoire parisien et le reste du pays.

Page 4 sur 10
} 
culture, structure encore la conception que ces mêmes agents se font de leur mission. On comprend ainsi que, si la diversité culturelle est prônée dans les domaines de la politique culturelle extérieure de la France, on peine à constater les effets d'une politique de diversité culturelle (qui pourrait constituer un objectif pour la décentralisation) à l'intérieur de nos frontières. Enfin, la politique des labels du ministère est aussi un obstacle à la mise en place de politiques culturelles territoriales. Il s'agit au contraire d'un outil de mise en équivalence des équipes, des lieux, des projets qui, de ce fait, tend à produire une (relative) homogénéité territoriale.

Les professionnels de la culture représentent le deuxième niveau d'acteurs impliqués dans les politiques culturelles territoriales. Ils semblent avoir maintenant dépassé la réserve généralisée dont ils ont longtemps fait preuve vis-à-vis de la déconcentration puis de la territorialisation. Il y a à cela plusieurs raisons : d'une part, les sources de financement public de la culture sont plutôt territoriales que centrales ; d'autre part, le champ des politiques culturelles s'est tellement ouvert depuis les années 80 que les demandes de soutien financier ont de meilleures chances d'aboutir quand elles sont présentées aux services des collectivités territoriales.

Les appréhensions des professionnels vis-à-vis du mouvement de territorialisation de la culture sont principalement liées au capital symbolique de l'État et à son pouvoir de consécration. L'État, ses services et agents ont encore aujourd'hui une certaine capacité à apporter une plus-value symbolique aux opérations qu'ils soutiennent, aux acteurs culturels qu'ils reconnaissent. Les mécanismes de production et de reproduction de ce «capital » sont assurément complexes. Le prestige du ministère n'est en effet pas seulement dû aux titres et compétences de celles et ceux qui travaillent pour lui. Ils résultent aussi des multiples actes d'allégeances que leur prodiguent leurs partenaires. Il n'en reste pas moins que cette forme d'échange de crédit d'Etat, qui traverse toute implication du ministère de la culture dans des actions de natures diverses (ce que P. Bourdieu appelait le travail de banquiers du capital symbolique de l'Etat), échappe encore nettement aux concurrences des collectivités territoriales. Le dédain du provincialisme est encore vivace dans les milieux artistiques, la presse régionale n'a pas, de son côté, le pouvoir de consécration de la presse parisienne dite nationale.

Mais, les appréhensions des professionnels de la culture naissent aussi d'une proximité redoutée avec le(s) politique(s). Quand le ministère développait la politique culturelle, celle-ci semblait relativement dégagée des enjeux politiques. Le rôle croissant des collectivités territoriales a, en revanche, rapproché les acteurs et les politiques culturelles des élus. Cela soulève deux difficultés souvent évoquées : le risque d'ingérence et de contrôle politique, d'une part, et son contraire, le risque d'absence de volonté politique des élus, d'autre part, lourd de menaces diverses pour des activités dépendant fortement des moyens publics. Les modalités de réduction de ces difficultés sont généralement trouvées au travers des financements croisés qui divisent les risques d'ingérence et favorisent une forme d'émulation entre partenaires publics.

\section{La territorialisation vue depuis les collectivités territoriales}

Les collectivités territoriales constituent, de façon globale, une troisième catégorie d'acteurs de la territorialisation. Si celle-ci est la résultante de leur rôle croissant dans les politiques culturelles, on peut penser qu'elle se traduit 
concrètement par une différenciation territoriale, elle-même croissante, des politiques culturelles. Or, la réalité montre que ce n'est pas nécessairement le cas.

On peut parler de territorialisation ${ }^{6}$ quand les problèmes culturels à traiter sont définis territoire par territoire, de même que les décisions prises pour y faire face. En ce sens, il y aurait territorialisation quand il y aurait une définition locale, territoriale, des problèmes publics et des mesures adoptées à leur sujet. On peut aussi parler de territorialisation quand le territoire devient l'objet de la politique publique, par opposition à d'autres objets comme le secteur (par exemple, culturel) ou une population particulière. Or, la définition territoriale des problèmes et des mesures n'est pas confirmée par l'étude de I'histoire des politiques culturelles. Entre 1975-80 et 1990 , les villes ont plutôt construit leurs politiques culturelles par empilement de rubriques en fonction de leurs moyens d'action et de leur volonté de développer ces politiques sans forcément s'appuyer sur des diagnostics très précis de la situation culturelle locale ${ }^{7}$. Les départements ont bénéficié de transferts de compétences, dans le champ des politiques culturelles et ont développé leur fonction traditionnelle de soutien des communes rurales. Sans mener de vraies politiques culturelles territoriales, ils ont construit des plans d'action appuyés sur des structurations intercommunales anticipant la loi du 13 août 2004 qui a imposé l'élaboration des schémas départementaux des enseignements artistiques du spectacle vivant. Les régions ont trouvé des secteurs d'intervention qui leur permettaient de se différencier des autres niveaux (communes et départements) mais sans procéder à une véritable territorialisation, c'est-à-dire sans s'attacher à un diagnostic culturel régional ni tenter de sortir des logiques sectorielles de traitement des questions culturelles.

Tout cela a abouti à une relative uniformisation des politiques culturelles territoriales. Des modèles de politiques culturelles pour les villes, les départements et les régions sont apparus et ont circulé entre les élus chargés de la culture et, surtout, entre les professionnels. Des normes d'équipements et d'actions ont été reproduites d'un territoire à un autre; les collectivités territoriales ont recouru aux mêmes outils pour servir leur rayonnement, les professionnels ont déployé sur tout le territoire national des façons d'être, de penser et de faire relativement uniformisées. Enfin, les demandes culturelles des classes moyennes cultivées se sont avérées elles aussi relativement uniformes alors qu'elles sont I'horizon d'attente le plus perceptible pour les responsables des politiques publiques de la culture. Le bilan de l'étude des dépenses des collectivités territoriales montre d'ailleurs, par catégorie de collectivité, des types assez précis de politiques culturelles ${ }^{8}$. C'est pourquoi, si la forme est celle de la territorialisation, dans le fond il s'agit plutôt d'une dé-territorialisation des politiques culturelles. Celles-ci se rapprochent de standards nationaux et donc d'un modèle vertical qui n'est pas imposé par l'État (bien incapable de le faire contrairement à ce que croient tant ses détracteurs que, parfois, ses « demandeurs »), mais qui résulte de l'action non concertée de tous ces acteurs. Cela s'explique en grande partie par l'absence d'outils de diagnostic territoriaux et culturels et par la politique de démocratisation culturelle qui fait que les territoires sont regardés à travers leurs manques et leurs écarts par rapport à des standards de politique culturelle qui visent à les rendre comparables les uns aux autres.

\footnotetext{
${ }^{6}$ Voir à ce sujet : A.C. Douillet, « Les élus ruraux face à la territorialisation de l'action publique », Revue Française de Science Politique, n4, 2004, pp583-606.

${ }^{7}$ E. Friedberg, PH. Urfalino, Le jeu du catalogue, La Documentation Française, 1984.

8 Ministère de la Culture et de la Communication, « Les dépenses culturelles des collectivités territoriales en $2002 »$, Les notes statistiques du DEPS, n²1, juillet 2006.

Page 6 sur 10
} 
Territorialiser signifie aussi que le territoire devient l'objet principal des politiques publiques, avec passage d'une logique sectorielle à une logique transversale. Or, les collectivités territoriales ont plutôt calqué le champ d'intervention de leurs services culturels sur le modèle de ceux de l'État en reproduisant ainsi la même définition (aussi imprécise soit-elle) du culturel qu'au niveau national. Elles ont, de cette façon, plus contribué à la sectorisation des affaires culturelles qu'à leur territorialisation. On observe de plus qu'elles ont souvent agi en faveur d'une égalisation de l'offre culturelle et, de cette manière, adhéré aux objectifs plus ou moins explicites des services de l'État. Les acteurs culturels professionnels sont souvent réticents à l'idée de logiques transversales auxquelles s'associeraient les politiques culturelles. Ils y voient une opposition entre une l'autonomie de leur champ (au sens sociologique du terme) et une logique extrinsèque, hétéronome, qui imposerait, de l'extérieur, d'autres objectifs aux activités artistiques et culturelles. Ils craignent ainsi ce qu'ils qualifient d'instrumentalisation de la culture, notamment au profit de politiques sociales, soit la réduction de leurs activités au rang d'outils au service d'autres fins. C'est ce que montre notamment leur faible investissement dans les débats concernant la construction de projets de territoire, en particulier dans le cas des grandes agglomérations urbaines.

Ainsi, la territorialisation est peut-être en œuvre (dans les rapports entre niveaux d'action publique), mais elle est encore peu perceptible dans le contenu des politiques culturelles (qui reste largement a-territorial, voire « déterritorialisé »). Précisons cependant, que les milieux culturels n'ont pas un comportement unanime sur ce sujet : la territorialisation suscite des craintes chez les plus « forts» (qui ont plus à perdre d'un changement de règles du jeu) et des espoirs de gains chez les plus « faibles ». Mais, d'une manière générale, les milieux culturels sont demandeurs d'une action de l'État; ils attendent qu'il fixe une règle et qu'il essaie de limiter les différenciations territoriales par ce moyen.

\section{L'intercommunalité culturelle au prisme de la territorialisation}

Les évolutions en matière culturelle de l'intercommunalité semblent en revanche s'opposer à la thèse selon laquelle les logiques territoriales aboutissent à une forme de déterritorialisation. L'intercommunalité a longtemps été un palliatif face à l'impossibilité de fusionner les 36700 communes de France; les premiers établissements de coopération intercommunale datent d'ailleurs de 1890 . La coopération entre les communes en milieu rural comme en milieu urbain, est devenue la règle, malgré les différences importantes qui peuvent exister entre les nouvelles institutions créées ${ }^{9}$. Pour les spécialistes, le transfert de la culture au profit de celles-ci était au départ une idée un peu saugrenue. Le ministère de I'Intérieur partageait ce point de vue: il avait considéré cette compétence comme optionnelle et l'avait concentrée sur des problématiques d'équipement.

\section{L'élan culturel intercommunal : triomphe de la territorialisation ?...}

Contrairement aux prévisions, la culture est pourtant très présente dans les compétences communautaires, celles des communautés d'agglomérations et de communes. Cette présence n'est pas toujours fondée sur des motivations effectives de politique culturelle, lorsqu'elle est associée au sport, par exemple; ou quand elle ne

\footnotetext{
${ }^{9}$ cf. E. Négrier, La question métropolitaine, PUG 2005. 
figure que de façon conservatoire, par impossibilité de retenir un nombre suffisant d'autres compétences optionnelles. Mais il existe souvent un véritable projet d'intérêt communautaire dans ce domaine, à vocation externe ou interne. En externe, la culture est sans doute un vecteur d'identification de territoires intercommunaux qui sont souvent nouveaux pour tout le monde. Au plan interne, elle fait partie des principes exprimés par les professionnels ou les élus pour agir ensemble, la culture devenant un instrument de la solidarité à l'échelle territoriale.

Les adversaires des politiques culturelles intercommunales sont des élus locaux des communes périphériques qui craignent de voir la ville-centre faire refinancer ses équipements par l'intercommunalité, mais ce sont aussi des responsables d'équipements culturels qui craignent le changement de tutelle administrative ou politique, les départements qui risquent de perdre de l'influence territoriale, etc. Fait paradoxal, les soutiens à l'intercommunalité culturelle viennent des mêmes protagonistes : des élus locaux périphériques sont heureux d'y recourir pour financer un équipement hors de portée des ressources municipales; des responsables de structures artistiques ont la possibilité d'élargir leurs interventions culturelles, des départements sont satisfaits de pouvoir cumuler des compétences diverses et complémentaires avec la métropole.

La situation varie d'un territoire à l'autre et les différences entre les territoires sont considérables. Aujourd'hui, la lecture publique, les enseignements artistiques et le spectacle vivant sont en tête des compétences intercommunales, ce qui s'explique par les vertus qui leur sont spécifiquement attribuées par les acteurs déterminants au moment des transferts: les élus locaux, mais aussi les acteurs culturels territoriaux dont le sens de l'action collective est plus prononcé. Pour qu'un territoire se projette dans la culture, on a, au fil du temps, identifié quelques ingrédients nécessaires. Il doit d'abord être dans un état d'esprit de solidarité territoriale, ce qui semble exclure les communautés de communes qui se sont constituées contre les communautés voisines, et qui partagent leur méfiance à l'égard du voisin et non un projet commun. Il doit aussi disposer d'un minimum de ressources économiques car le montant de la dotation bonifiée de l'État dépend des transferts possibles du niveau communal au niveau intercommunal. Le leadership, son caractère plus ou moins stable et coopératif, constituent une troisième condition. Enfin, l'implication des acteurs culturels au concret dans le portage des projets, a une influence sur la faisabilité des transferts, et sur leurs conditions de mise en œuvre.

Il existe aujourd'hui au moins quatre ou cinq modèles d'intercommunalité culturelle. Le premier est celui de la culture cosmétique, avec un transfert de compétences et une ligne budgétaire inexistants ou presque. Le deuxième est celui de la vision rayonnante de la compétence culturelle, avec le choix d'une intercommunalité seule responsable des équipements qui rayonnent au-delà du municipal. Le troisième modèle affecte le maximum de ressources à un domaine test avant de transférer les données de l'expérience à d'autres domaines (exemple : le transfert de toutes les actions et équipements de lecture publique). Le quatrième modèle est celui de la maximisation financière (tous les équipements, quelque soit leur envergure) car le montant de la dotation bonifiée de l'État est fonction du nombre des équipements transférés. Le dernier modèle est celui du « tout culturel » : tout est transféré à l'intercommunalité y compris les subventions aux associations culturelles municipales. Derrière ces types idéaux se profilent des processus qui sont fortement distincts d'un lieu à l'autre. 


\section{... ou consécration des standards professionnels?}

Il reste cependant à distinguer des différenciations territoriales à l'œuvre, notamment dans les processus politiques intercommunaux, et des formes de mimétismes qui, simultanément, peuvent se faire jour dans le contenu même de ces politiques culturelles, à rebours donc des promesses de territorialisation.

La loi Chevènement n'est évidemment pas appliquée au pied de la lettre ni de la même façon partout en France et il existe des différences évidentes de mise en œuvre de cette loi selon les secteurs d'intervention et selon les territoires. Au niveau des communes, puis des départements et enfin des régions, les politiques culturelles ne se sont pas développées d'un même élan sur l'ensemble du territoire national. Les différences observables aujourd'hui entre intercommunalités «ressemblent » aux différences qui pouvaient apparaître, plus nettement qu'aujourd'hui, entre des communes de même taille, départements ou régions. Si, en raison de leurs héritages, de leurs situations économiques, sociales, géographiques et politiques, des différences marquent I'action culturelle de collectivités de même nature, elles ne contredisent pas un diagnostic soulignant l'apparition de contenus relativement standards. Il en va donc de même des intercommunalités qui, dans leurs champs de compétences, déploient des politiques dont on peine à dire en quoi elles s'écarteraient des référentiels d'action qui sont à l'œuvre pour les mêmes secteurs (lecture publique, enseignement musical, etc.) au sein des communes. Plus généralement, le modèle français de politique culturelle réapparaît au niveau des structures intercommunales. Il se caractérise par un financement essentiellement public, par un souci de professionnalisation de la vie culturelle y compris dans les secteurs à fort taux de bénévolat, par une logique verticale descendante (porter la culture dans les territoires et auprès de populations qui en sont dépourvus ou éloignés) et assez souvent par une conception essentiellement esthétique de la culture. Parfois, l'intercommunalisation d'un secteur culturel (l'enseignement musical, la lecture publique, en particulier) est l'occasion, localement, d'une transformation substantielle de l'action antérieure. Mais il s'agit généralement de mettre en œuvre des orientations débattues depuis plusieurs décennies dans les milieux professionnels concernés et déjà appliquées au niveau de territoires comparables, voire de communes ou de départements.

La différenciation territoriale des contenus de politiques culturelles serait aussi plus nette si l'on pouvait observer des actions visant à rendre la vie culturelle plus autonome vis-à-vis des pouvoirs publics, ne faisant pas de la professionnalisation à la fois un objectif et une condition des soutiens publics, essayant de travailler à partir de toutes les richesses d'un territoire et traitant de la culture en son sens anthropologique (notamment présent dans les textes de I'UNESCO concernant la diversité culturelle) et non pas au sens plus restreint des œuvres de l'art et de l'esprit. On observe, enfin, que la décentralisation n'aurait guère d'intérêt si elle ne favorisait pas une plus forte mise en débat des choix publics (notamment culturels). La relative proximité entre autorités politiques et citoyens est en effet potentiellement riche d'une capacité, quasi inexistante au niveau national, de délibérations ouvertes sur la définition du bien commun. Or, la politique culturelle en France a été soustraite du débat public : elle est peu débattue au Parlement et l'action culturelle a fonctionné de façon très personnalisée dans le schéma classique de la $V^{\mathrm{è}}$ République. Elle s'est ainsi gagné l'adhésion des grands professionnels de la culture, consacrés par le pouvoir d'État et qui voyaient grâce à lui les activités culturelles mises à l'abri d'un débat politique qui aurait pu les mettre en cause. Mais aujourd'hui, le débat sur la définition des politiques culturelles est devenu nécessaire. Leurs difficultés, leur crise, 
leurs nouveaux enjeux (le développement local, les différences culturelles, l'interculturalité, l'impact des nouvelles technologies sur la production artistique et sa diffusion, etc.) imposent des réflexions et des décisions dont la qualité tant technique que politique, sera mieux assurée si elles sont largement ouvertes. De ce point de vue, la territorialisation est un facteur favorable permettant de produire un diagnostic partagé, d'inventer des mesures nouvelles et de donner ainsi un sens plus politique à la territorialisation. Il serait d'ailleurs curieux qu'à l'échelle locale on soit longtemps encore rétif à la mise en débat des politiques culturelles, alors que I'Union européenne, en adoptant en 2007 l'agenda pour la culture proposée par la Commission, a aussi fait le choix de renforcer, via un forum culturel européen, son dialogue avec les organisations de la société civile concernées par ces enjeux.

La territorialisation des politiques culturelles est aujourd'hui moins un projet politique qu'un mouvement permanent et polymorphe (centralisation et décentralisation, déconcentration, changements d'échelle ascendants et descendants...). Alors que jadis, les enjeux locaux étaient secondaires et spécifiques, ils s'interpénètrent aujourd'hui avec les enjeux dits centraux. L'action territoriale est devenue «banale », fondée sur des logiques professionnelles largement partagées, par exemple.

C'est peut-être pour cela que, indépendamment des plus-values apportées aux politiques culturelles par l'intercommunalité et, plus largement, par les changements politiques et institutionnels constitutifs de la territorialisation, on n'observe pas à cette occasion une transformation profonde des types de politiques culturelles qui sont à l'œuvre en France depuis plusieurs décennies. La territorialisation des processus ayant en ce domaine sensiblement devancé celle des contenus. Cela lui permet d'être aux avant-postes d'un débat politique nécessaire, sans pour autant incarner la rupture. 